\title{
EFICÁCIA DA SOLUÇÃO REVELADORA DE LINFONODOS EM PEÇAS DE GASTRECTOMIA COM LINFADENECTOMIA POR ADENOCARCINOMA GÁSTRICO
}

\section{EFFICACY OF LYMPH NODES REVEALING SOLUTION IN GASTRECTOMY WITH LYMPHADENECTOMY SPECIMENS FROM GASTRIC CARCINOMA}

\author{
Pablo Rodrigo de Siqueira ${ }^{1}$ \\ Sidney Roberto Nadal, TCBC-SP ${ }^{2}$ \\ Giulio Cesare Santo ${ }^{3}$ \\ Marcos Mota da Silva ${ }^{4}$ \\ Francisco César Martins Rodrigues, TCBC-SP ${ }^{2}$ \\ Carlos Alberto Malheiros, TCBC-SP ${ }^{1}$
}

\begin{abstract}
RESUMO: Com o intuito de identificar um número maior de linfonodos nas peças cirúrgicas de gastrectomia com linfadenectomia por adenocarcinoma gástrico, utilizamos a solução reveladora de linfonodos (SRL), após a retirada dos linfonodos identificados pelo método tradicional de visualização e palpação, em 13 doentes. Encontramos 222 nódulos linfáticos (17,1 por doente) pela maneira habitual e, depois, aplicando a SRL, retiramos mais 123, ou seja, 9,5 (55,5\%) de linfonodos a mais por doente. A histologia mostrou 39 linfonodos com invasão neoplásica entre os encontrados com o método tradicional e oito com a SRL. O diâmetro dos nódulos identificados com a SRL foi menor que o dos detectados com o outro método. O estádio da doença não se modificou após a análise dos linfonodos encontrados com a SRL. Concluímos, pela análise estatística dos dados, que a SRL é eficaz para identificar linfonodos não detectados pelo método tradicional.
\end{abstract}

Unitermos: Câncer gástrico. Linfonodos. Solução reveladora de linfonodos. Gastrectomia.

\section{INTRODUÇÃO}

O câncer gástrico é enfermidade com distribuição global e o neoplasma maligno de maior prevalência no sexo masculino e o segundo entre as mulheres, em nosso meio ${ }^{1}$. Embora haja declínio do número de casos nos últimos anos, é a causa mais comum de óbito por doença maligna no mundo ${ }^{2}$. Entretanto, sua distribuição geográfica não é homogênea. A taxa de letalidade é cinco a nove por 100.000 nos países com as menores incidências (Austrália e Estados Unidos), enquanto nos de grande prevalência (Japão e
Chile) é superior a 30 por 100.000 habitantes $^{3}$. Isso justifica o grande investimento, principalmente no Japão, para que o diagnóstico seja feito nas fases mais iniciais, visto que o sucesso do tratamento depende do estádio da doença ${ }^{4}$. A eficácia dos métodos de rastreamento naquele país é reconhecida, uma vez que $30 \%$ a $40 \%$ desses tumores são precoces no momento da detecção. Já nos países ocidentais, $65 \%$ dos doentes encontram-se nas fases mais avançadas dessa neoplasia e apenas $6 \%$ a $16 \%$ no estádio $\mathrm{I}^{5}$.

Os procedimentos radiológicos e principalmente os endoscópicos sugerem o diagnóstico. Os métodos de ima-

${ }^{1}$ Residente do Departamento de Cirurgia da FCMSCSP.

${ }^{2}$ Doutores em Cirurgia Geral da FCMSCSP.

${ }^{3}$ Médico Patologista da FCMSCSP

${ }^{4}$ Residente de $3^{\circ}$ ano do Departamento de Ciências Patológicas da FCMSCSP

Recebido em 17/5/99

Aceito para publicação em 13/6/2000

Trabalho realizado na Área V - Grupo de Estômago e Duodeno do Departamento de Cirurgia e no Departamento de Ciências Patológicas da Faculdade de Ciências Médicas da Santa Casa de São Paulo — FCMSCSP 
gem, tais como a ultra-sonografia, a tomografia computadorizada e, na atualidade, a ultra-sonografia endoscópica têm contribuído para estabelecer o estádio das lesões ${ }^{6}$. A ultra-sonografia endoscópica tem sensibilidade de $85 \%$ para revelar a invasão do tumor na parede gástrica ${ }^{7}$ e acurácia entre $65 \%$ e $87 \%$ para avaliar a presença de linfonodos metastáticos ${ }^{8}$, considerados os principais fatores prognósticos ${ }^{9}$. Os estádios determinados pela Union Internacional de Controle du Cancer (UICC) e pela American Joint Cancer Committee (AJCC) baseiam-se na análise desses parâmetros e consideram ainda a disseminação metastática visceral ${ }^{10}$. A Japanese Research Society for Gastric Cancer (JRSGC), além desses, avalia também a localização do tumor no estômago ${ }^{6}$.

O tratamento mais eficaz é a gastrectomia ${ }^{10}$ com extirpação linfonodal extensa, que pode prolongar o tempo de sobrevivência ${ }^{6}$, evitando a doença residual em linfonodos comprometidos e de outra forma não extirpados. A determinação correta do estádio do tumor, no período pósoperatório, é importante não só para a definição do prognóstico do doente, como também para a indicação de terapêutica complementar. Para isso, são desejáveis a identificação e a análise anatomopatológica do maior número possível de linfonodos na peça cirúrgica.

O método tradicional para procura de nódulos linfáticos na peça consiste na visualização direta e palpação. Entretanto, os linfonodos muito pequenos raramente são encontrados. Recentemente Koren et al. ${ }^{11}$ empregaram a "solução reveladora de linfonodo" por eles criada, composta por $65 \%$ de etanol a $95 \%, 20 \%$ de dietil-éter, $10 \%$ de formol tamponado e $5 \%$ de ácido acético glacial, na identificação linfonodos de peças cirúrgicas de adenocarcinoma de cólon. Nela, após seis horas de imersão, os linfonodos tornam-se esbranquiçados e destacam-se da gordura amarelada, facilitando a identificação de nódulos com até $0,5 \mathrm{~mm}^{11}$.
A necessidade de definir melhor o comprometimento linfonodal dos neoplasmas malignos do estômago, e desta maneira o estádio correto da doença, nos estimulou a realizar este estudo prospectivo para avaliar a efetividade da SRL na identificação dos linfonodos, nas peças cirúrgicas de gastrectomias por adenocarcinoma gástrico, do que não encontramos relato na literatura consultada.

\section{PACIENTES E MÉTODOS}

Estudaram-se as peças cirúrgicas de 13 pacientes com adenocarcinoma gástrico operados, pela Área de Estômago e Duodeno do Departamento de Cirurgia da Faculdade de Ciências Médicas da Santa Casa de São Paulo, no período de novembro de 1997 a julho de 1998.

Destes, seis eram do sexo masculino e sete do sexo feminino com idades entre 33 e 73 anos e média de 55,2 anos. Sete apresentavam tumor no antro, quatro no corpo e em dois a lesão acometia tanto o antro como o corpo gástrico. Doze foram submetidos à gastrectomia subtotal e um à gastrectomia total, com linfadenectomia (Tabela 1).

Enviamos as peças cirúgicas, com os grupos linfonodais previamente separados de acordo com a sistematização da JRSGC, ao Laboratório de Anatomia Patológica. Inicialmente, retiraram-se os linfonodos identificados conforme a maneira tradicional ( visualização direta e palpação). Isso feito, o restante da peça cirúrgica foi imersa na SRL durante seis horas. Passado esse período, lavamos em água corrente e seccionamos os tecidos, com bisturi, a intervalos entre 2 e $3 \mathrm{~mm}$, e os linfonodos encontrados foram retirados

Mediu-se o maior diâmetro com paquímetro. Após a mensuração, incluíram-se os linfonodos em parafina, feitos cortes finos, coloração pela hematoxilina-eosina, e as lâminas examinadas à microscopia óptica para avaliar a presença de neoplasia.

Tabela 1

Distribuição de 13 doentes submetidos a gastrectomias por neoplasma maligno do estômago conforme sexo, idade, localização do tumor, operação realizada, número de linfonodos retirados, método de identificação e estádio da doença. Sta Casa de São Paulo. 1998

\begin{tabular}{|c|c|c|c|c|c|c|c|c|c|c|c|}
\hline \multirow{2}{*}{ Iniciais } & \multirow{2}{*}{ Sexo } & \multirow{2}{*}{ Idade } & \multirow{2}{*}{ Local } & \multirow{2}{*}{ Operação } & \multirow{2}{*}{\multicolumn{3}{|c|}{$\begin{array}{l}\text { Linf. Met. Trad } \\
\text { s/ met c/met Total }\end{array}$}} & \multicolumn{3}{|c|}{ Linf c/SRL } & \multirow{2}{*}{ Estádio } \\
\hline & & & & & & & & s/ met & / met & Total & \\
\hline FAP & masc & 55 & antro & gastrect parcial & 08 & 03 & 11 & 12 & 00 & 12 & T3N1M1 \\
\hline MOS & fem & 41 & antro & gastrect subtotal & 01 & 02 & 03 & 06 & 00 & 06 & $\mathrm{~T} 2 \mathrm{~N} 1 \mathrm{M} 0$ \\
\hline ALS & fem & 58 & antro & gastrect subtotal & 14 & 02 & 16 & 16 & 00 & 16 & $\mathrm{~T} 2 \mathrm{~N} 1 \mathrm{M} 1$ \\
\hline BPDC & fem & 72 & corpo/antro & gastrect total & 02 & 05 & 07 & 02 & 01 & 03 & T3N1M0 \\
\hline GDG & masc & 52 & antro & gastrect subtotal & 16 & 15 & 31 & 06 & 00 & 06 & T4N2M0 \\
\hline RJSA & masc & 56 & antro & gastrect subtotal & 24 & 01 & 25 & 07 & 01 & 08 & $\mathrm{~T} 2 \mathrm{~N} 1 \mathrm{M} 0$ \\
\hline JMM & masc & 63 & antro & gastrect subtotal & 28 & 00 & 28 & 16 & 00 & 16 & T2N0M0 \\
\hline $\mathrm{EF}$ & fem & 42 & antro & gastrect subtotal & 03 & 03 & 06 & 08 & 03 & 11 & T4N1M1 \\
\hline RRS & fem & 63 & corpo & gastrect subtotal & 20 & 00 & 20 & 05 & 00 & 05 & T1N0M0 \\
\hline MMS & fem & 54 & corpo & gastrect subtotal & 17 & 04 & 21 & 13 & 02 & 15 & $\mathrm{~T} 2 \mathrm{~N} 1 \mathrm{M} 0$ \\
\hline VXC & masc & 56 & corpo & gastrect subtotal & 15 & 02 & 17 & 05 & 00 & 05 & T2N1M0 \\
\hline SPS & fem & 33 & corpo & gastrect subtotal & 14 & 02 & 16 & 03 & 01 & 04 & $\mathrm{~T} 2 \mathrm{~N} 1 \mathrm{M} 0$ \\
\hline FAS & masc & 73 & corpo/antro & gastrect subtotal & 21 & 00 & 21 & 16 & 00 & 16 & T2N0M0 \\
\hline
\end{tabular}

Linf. Met. Trad. $=$ Linfonodos Identificados Pelo Método Tradicional .

Linf. c/ SRL = Linfonodos Identificados pela SRL. 
Determinamos o estádio do tumor pela classificação TNM, segundo a UICC (Tabela 2), em duas etapas: com o resultado histopatológico dos linfonodos identificados pelo método tradicional e, numa segunda fase, somando a esses, o dos linfonodos retirados com a SRL.

Para determinar a efetividade da SRL, comparamos o número de linfonodos encontrados da maneira tradicional, com a soma total dos revelados por ambos os métodos.

Submetemos os dados obtidos à análise estatística pelo método do qui-quadrado e do t de Student, aplicando o nível de significância de 5\%.

\section{RESULTADOS}

Com o método tradicional, retiramos 222 linfonodos das 13 peças cirúrgicas (média de 17,07 por paciente). Com

Tabela 2

Classificação TNM/R de acordo com a UICC 1997

\begin{tabular}{|c|c|}
\hline Categoria $\mathrm{T}$ & \\
\hline Tis & $\begin{array}{l}\text { Limitado à mucosa, sem penetração na } \\
\text { membrana basal }\end{array}$ \\
\hline T1a & $\begin{array}{l}\text { Limitado à mucosa (invasão da lâmina } \\
\text { própria) }\end{array}$ \\
\hline $\mathrm{T} 1 \mathrm{~b}$ & Tumor invade submucosa \\
\hline $\mathrm{T} 2 \mathrm{a}$ & Tumor invade muscular própria \\
\hline $\mathrm{T} 2 \mathrm{~b}$ & Tumor invade subserosa \\
\hline T3 & $\begin{array}{l}\text { Penetração da serosa sem invasão de tecido } \\
\text { adjacente }\end{array}$ \\
\hline $\mathrm{T} 4$ & Tumor invade tecido adjacente e/ou órgãos \\
\hline \multicolumn{2}{|r|}{ 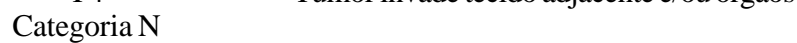 } \\
\hline No & Sem envolvimento linfonodal \\
\hline $\mathrm{N} 1$ & De um a seis linfonodos envolvidos \\
\hline $\mathrm{N} 2$ & De sete a 15 linfonodos envolvidos \\
\hline N3 & Mais que 15 linfonodos envolvidos \\
\hline Proporção N & $\begin{array}{l}\text { Proporção entre linfonodos envolvidos e } \\
\text { removidos }\end{array}$ \\
\hline \multicolumn{2}{|l|}{ Categoria M } \\
\hline M0 & Sem metástase a distância \\
\hline M 1 & Metástase a distância \\
\hline \multicolumn{2}{|l|}{ Categoria R } \\
\hline R0 & Sem tumor residual \\
\hline R1 & Tumor residual microscópico \\
\hline $\mathrm{R} 2$ & Tumor residual macroscópico \\
\hline
\end{tabular}

o uso da SRL encontramos mais 123 (média de 9,46 por paciente), aumentando a média para 26,53, ou 55,5\% a mais. A análise estatística mostrou que a SRL foi efetiva na identificação de linfonodos $(\mathrm{p}=0,022)$ (Tabela 3 ).

O diâmetro dos 222 nódulos linfáticos descobertos com o método tradicional oscilou entre um e $30 \mathrm{~mm}$, com média de 4,56mm. Os 123 linfonodos encontrados após o emprego da S.R.L eram menores, apresentando diâmetro médio de $2,71 \mathrm{~mm}$, variando entre um e $20 \mathrm{~mm}(\mathrm{p}=0,000)$ (Tabela 4).

Dos encontrados com o método tradicional, 183 linfonodos não eram metastáticos (negativos) e 39 apresentavam invasão neoplásica (positivos). Entre aqueles revelados pela SRL, 115 eram negativos e oito positivos (Tabela 3). A análise estatística destes dados sugere que o método tradicional revelou mais linfonodos metastáticos do que o da SRL $(p=0,007)$. Os linfonodos negativos pelo primeiro apresentavam diâmetro médio de $3,91 \mathrm{~mm}$, variando de um a $20 \mathrm{~mm}$, semelhante aos positivos que mediram entre um e $30 \mathrm{~mm}$, com média de $7,58 \mathrm{~mm}$ ( $\mathrm{p}=0,608)$. Porém, a comparação entre o diâmetro médio dos linfonodos negativos e positivos do método da SRL revelou que os positivos eram maiores $(\mathrm{p}=0,025)$ (Tabela 4).

A maioria dos linfonodos negativos identificados com o método tradicional $(69,94 \%)$ apresentou diâmetro entre um e $4 \mathrm{~mm}$, enquanto $56,41 \%$ dos positivos mediram entre 5 a $10 \mathrm{~mm}$ (Tabela 5). Cerca de $91,38 \%$ dos nódulos negativos detectados após o uso da S.R.L apresentaram diâmetro entre $1 \mathrm{a} 4 \mathrm{~mm}$, e dos positivos $50 \%$ tinham entre $1 \mathrm{e}$ $4 \mathrm{~mm}$ e os outros $50 \%$ entre 5 e $10 \mathrm{~mm}$. (Tabela 6 ).

O estádio dos tumores permaneceu o mesmo em todos os doentes antes e após a identificação dos linfonodos pela SRL.

\section{DISCUSSÃO}

Rother et al. ${ }^{12}$; Lee et al. ${ }^{13}$; Isozaki et al..${ }^{14} \mathrm{e}$ Makino et al. ${ }^{15}$ relataram a importância de realizar a linfadenectomia e avaliar a presença de invasão tumoral nos linfonodos extirpados mostrando que o número dos metastáticos está relacionado às taxas de sobrevivência. De acordo com Okusa et al. ${ }^{16}$ seria de $81 \%$ na ausência de metástase linfonodal, $63 \%$ para pacientes com um a três linfonodos comprometidos, $47 \%$ para aqueles com quatro a seis e $29 \%$

Tabela 3

Distribuição de 222 linfonodos identificados pelo método tradicional e 123 revelados pela SRL, em peças cirúrgicas de gastrectomias por neoplasma maligno do estômago. Sta Casa de São Paulo, 1998

\begin{tabular}{|c|c|c|c|c|}
\hline $\begin{array}{c}\text { Método de } \\
\text { identificação }\end{array}$ & Linfonodos negativos & Linfonodos positivos & Total & $\begin{array}{l}\text { Número médio } \\
\text { de linf/doente }\end{array}$ \\
\hline Tradicional $\dagger$ & 183 & 39 & 222 & $17,07 \pm 8,54^{*}$ \\
\hline $\mathrm{SRL} \dagger$ & 115 & 08 & 123 & $9,46 \pm 5,04$ \\
\hline Total & 298 & 47 & 345 & $26,53 \pm 10,93 *$ \\
\hline
\end{tabular}

* t de Student: comparando o número médio de linfonodos/doente dos grupo Tradicional e Total p = 0,022 (significante)

$\dagger$ Qui-quadrado: comparando o número de linfonodos positivos e negativos dos grupos Tradicional e SRL $\mathrm{m}^{2}=0,007$ (significante) 
Tabela 4

Distribuição conforme o diâmetro médio e a presença ou não de metástases em 222 linfonodos identificados pelo método tradicional e 123 revelados pela SRL, em peças cirúrgicas de gastrectomias por neoplasma maligno do estômago. Sta Casa de São Paulo, 1998

\begin{tabular}{l|c|c|r}
\hline \multirow{2}{*}{ Método de identificação } & \multicolumn{3}{|c}{ Diâmetro médio dos linfonodos em mm } \\
\cline { 2 - 4 } & Negativos & Positivos & Total \\
\hline Tradicional & $3,91 \pm 2,87^{*}$ & $7,58 \pm 6,54^{*}$ & $4,56 \pm 3,99 \dagger$ \\
SRL & $2,56 \pm 2,11 \#$ & $4,62 \pm 2,26 \#$ & $2,71 \pm 2,17 \dagger$ \\
\hline
\end{tabular}

$\dagger \mathrm{p}=0,000$ (significante).

$* \mathrm{p}=0,608$ (não significante).

$\# \mathrm{p}=0,025$ (significante).

Tabela 5

Distribuição de 222 linfonodos identificados pelo método tradicional em peças cirúrgicas de gastrectomias por neoplasma maligno do estômago, conforme o diâmetro e a presença de neoplasia. Sta. Casa de São Paulo, 1998

\begin{tabular}{|c|c|c|c|c|c|c|}
\hline \multirow{2}{*}{$\begin{array}{l}\text { Diâmetro } \\
(\mathrm{mm})\end{array}$} & \multicolumn{2}{|c|}{ Linfonodos negativos } & \multicolumn{2}{|c|}{ Linfonodos positivos } & \multicolumn{2}{|c|}{ Total } \\
\hline & № & $\%$ & № & $\%$ & № & $\%$ \\
\hline 1 a 4 & 128 & 69,94 & 11 & 28,20 & 139 & 62,61 \\
\hline 5 a 10 & 46 & 25,13 & 22 & 56,41 & 68 & 30,63 \\
\hline$>11$ & 09 & 4,91 & 06 & 15,38 & 15 & 6,76 \\
\hline Total & 183 & 100 & 39 & 100 & 222 & 100 \\
\hline
\end{tabular}

Tabela 6

Distribuição de 123 linfonodos identificados pela SRL em peças cirúrgicas de gastrectomias por neoplasma maligno do estômago, conforme o diâmetro e a presença de neoplasia. Sta Casa de São Paulo, 1998

\begin{tabular}{|c|c|c|c|c|c|c|}
\hline \multirow{2}{*}{$\begin{array}{c}\text { Diâmetro } \\
(\mathrm{mm})\end{array}$} & \multicolumn{2}{|c|}{ Linfonodos negativos } & \multicolumn{2}{|c|}{ Linfonodos positivos } & \multicolumn{2}{|c|}{ Total } \\
\hline & № & $\%$ & № & $\%$ & № & $\%$ \\
\hline $1 \mathrm{a} 4$ & 105 & 91,30 & 04 & 50 & 109 & 88,62 \\
\hline 5 a 10 & 09 & 7,82 & 04 & 50 & 13 & 10,57 \\
\hline$>11$ & 01 & 0,86 & 00 & 00 & 01 & 0,81 \\
\hline Total & 115 & 100 & 08 & 100 & 123 & 100 \\
\hline
\end{tabular}

quando mais de seis, após cinco anos. Rhoder et al. ${ }^{12}$ afirmaram que a proporção entre nódulos comprometidos e examinados seria desfavorável quando maior do que 0,2 .

O diagnóstico histopatológico de pN0 (ausência de comprometimento linfonodal) requer a linfadenectomia regional e exame histológico de no mínimo 15 linfonodos, retirados da peça cirúrgica, número considerado adequado para determinar o estádio correto da doença, segundo Wells ${ }^{7}$. Em nossa casuística encontramos a média de 26,5 por doente. Conforme citado por Lee et al. ${ }^{13}$ ressecções que incluam mais que 60 linfonodos não acrescentam informação significativa; definiram também que a extirpação rotineira com média de 30 ou mais linfonodos pode ser adotada como controle de qualidade cirúrgica. Esses números tiveram como base a avaliação de peças cirúrgicas de gastrectomia total. Em nosso estudo, 12/13 gastrectomias fo- ram subtotais, conseqüentemente, com menor número de linfonodos extirpados. Acreditamos que os parâmetros citados por Lee et al. ${ }^{13}$ poderão ser alcançados com a SRL se trabalharmos apenas com peças cirúrgicas de gastrectomia total.

Entretanto, além do cirurgião dissecar e extirpar a quantidade adequada de linfonodos, é necessário que o patologista retire e avalie o maior número possível de gânglios na peça cirúrgica. Com o método tradicional, nem sempre encontramos os nódulos muito pequenos. Vários autores propuseram protocolos sobre o clareamento da gordura na peça operatória, para identificá-los $17,18,19,20,21$. No entanto, têm custo elevado, são necessários até sete dias para o processo, além da necessidade de profissionais especializados ${ }^{17,18,19,20,21}$. A substância que empregamos (SRL) tem preço calculado de um real para cada litro, pode 
ser usada por mais de uma vez e o procedimento demora seis horas.

Aplicando a SRL, encontramos mais 9,5 linfonodos por peça ressecada, à semelhança do estudo de Koren et al. ${ }^{11}$ que, com a mesma solução em peças de colectomia por adenocarcinoma de cólon, retiraram mais 8,6, em média. Esses autores identificaram o triplo de linfonodos com a SRL, e nós 55,5\% a mais. Entretanto, eles estudaram doentes portadores de tumores de cólon. Não encontramos trabalhos em que utilizaram a SRL em neoplasmas malignos do estômago. O diâmetro dos linfonodos identificados com a SRL variou de 1 a $11 \mathrm{~mm}$, sendo que $86,6 \%$ destes apresentavam tamanho menor que $5 \mathrm{~mm}$, resultados também comparáveis ao desse autores ${ }^{11}$. Os linfonodos positivos revelados pela SRL eram maiores do que os sem metástases, entretanto, houve linfonodos de 1 e $2 \mathrm{~mm}$ com invasão neoplásica.

Não observamos alteração do estádio da doença levando em conta os resultados do método da SRL devido, provavelmente, ao pequeno número de casos estudados e também porque a maioria dos pacientes estava nas fases mais avançadas da enfermidade. Pretendemos aplicar o método em tumores precoces para avaliarmos melhor esse aspecto. Todavia, três doentes ficaram no limite superior da classificação N1, por apresentarem seis linfonodos metastáticos. No relato de Koren et al. ${ }^{11}$, o único publicado, houve agravamento do estádio em um terço dos doentes portadores de tumores do cólon. Acreditamos, a exemplo desses autores, que a determinação correta do estádio, possibilitaria aumento da sobrevivência desses pacientes, pois, em alguns deles, com essa mudança, a terapêutica adjuvante estaria indicada.

O método da SRL é barato, simples, rápido e de fácil execução. Mostrou-se efetivo para revelar nódulos linfáticos adicionais em peças de gastrectomia por adenocarcinoma do estômago. Acreditamos que a substância possa ser utilizada para identificar linfonodos em peças provenientes de extirpação de outros tumores malignos. Isso possibilitaria definir o estádio correto dessas neoplasias e, se for o caso, instituir a terapêutica neoadjuvante, bem como para conhecermos o prognóstico da doença.

A partir dos resultados encontrados nas condições de realização do presente trabalho, concluímos que a SRL é eficaz na identificação de linfonodos não evidenciados pelo método tradicional em peças cirúrgicas de gastrectomias por adenocarcinoma gástrico.

\begin{abstract}
In spite of it's decreasing incidence in the last years, gastric cancer remains the most frequent cause of death by malignant. Prognosis depends on gastric wall tumor invasion, lymph node involvement and distant metastasis. Small lymph nodes (less than $1 \mathrm{~mm}$ ) are rarely identified, and, disease status and prognosis could change if some are metastatic. To identify lymph nodes in gastrectomy and lymphadenectomy specimens from gastric cancer, we have used a lymph node revealing solution (LNRS) after having taken the visible and palpable lympg nodes off. We have found 222 nodes with this technique (17.1 per patient) and more 123 nodes with LNRS (9.5 per patient), 55.5\% additional nodes. Biopsies have shown 39 metastatic nodes revealed by the traditional method and eight with LNRS. Statistics showed that LNRS was effective in lymph nodes detection. LNRS revealed nodes were smaller. Disease status did not change after addiction of LNRS detected nodes. We concluded LNRS was effective in lymph nodes searching from the studied specimens.
\end{abstract}

Key words: Gastric cancer. Lymph nodes. Lymph nodes revealing solution. Gastrectomy.

\section{REFERÊNCIAS}

1. Domene EC, Volpe P, Onari P et al. Gastrectomia Subtotal com linfadenectomia a DII por videolaparoscopia. In, Domene CE, Chaib E, Silva JH. Esôfago Hoje e Gastrão 1997;12(supl 1):160-162.

2. Rosin RD. Tumors of the Stomach. In, Zinner MJ. Maingot's Abdominal Operations. $10^{\text {th }}$ ed, Editora Appleton e Lange, 1997.

3. Bresciani CJC, Rodrigues JJG, Pinotti HW. Câncer Gástrico. In, Fundamentos de Gastroenterologia Cirúrgica, 1 a ed. São Paulo. Editora Contexto, 1990.

4. Meyer HJ, Jayne J, Wilke H.Perspectives of Surgery and Multimodality Treatment in Gastric Garcinoma, J Cancer Res Clin Oncol 1992; 119: 384-394.
5. Tanton G. Adjuvant Treatment in Gastric Cancer. Possibility to take or to leave it. Acta Chir Belg 1996; 96: 59-61.

6. Pinotti HW. Câncer do Estômago. Roteiro de trabalho para o cirurgião. FMUSP, 1997.

7. Wells S A. Gastric Cancer. Curr. Probl. Surg 1997: 34: 842845.

8. Rösch Th. Endosonographic staging of gastric cancer: a review of literatures results. Gastrointest Clin North Am 1995; 3: 549-57.

9. Yu CC et al. Pathological prognostic factors in the second British Stomach Cancer Group. Br J Surg 1995; 71: 1106-1110.

10. Gonçalves AJ, Nadal SR, Malheiros CA et al. Tratamento cirúrgico do câncer gástrico. Análise de 254 casos. Arq Med Hosp Fac Sta Casa S Paulo 1989;9:64:69. 
11. Koren R et al. Lymph node - revealing solution. Simple new method for detecting minute lymph nodes in colon carcinona. Dis Colon Rectum 1997; 40: 407-410.

12. Rodher ID, Bottcher K, Siewert JR et al. Prognostic factors in gastric carciona. Cancer 1993; 72: 2089-97.

13. Lee WJ, Lee PH, Yue SC et al. Lymph node metastases in gastric cancer: significance of positive number. Oncology 1995; 52: 45-50.

14. Isozaki H, Okagima $\mathrm{K}$, Kawashima y et al. Prognostic value of the number of metastatic limph node in gastric cancer whit radical surgery. J Surg Oncol 1993; 53: 247-251.

15. Makino M, Moriwaki 5, Yonekawa M et al. Prognostic significance of the number or metastatic lymph node in pacientes with gastric cancer. J Surg Oncol 1991; 47: 12-16.

16. Okusa T, Nakane y, Boku T et al. Quantitative analysis of nodal involvement with respect to survival rate after curative gastrectomy for carcinoma. Surgery 1996; 170: 488-94.

17. Cawthorn SJ, Gibbs NM, Marks CG. Clearence technique for the detection of lymph nodes in colorectal cancer. Br J Surg 1986;73: 58-60.

18. Herrera-Ornelas L, Justiniano J, Castillo $\mathrm{N}$ et al. Metastases in small lymph nodes from colon cancer. Arch Surg 1987;122: 1253-1256.
19. Scott KWM, Grace RH. Detection of lymph node metastases in colorectal carcinoma before and after clearence. Br J Surg 1989;76: 1165-1167.

20. Hida J, Mori N, Kubo, R et al. Metastases from carcinoma of the colon and rectum detected in small lymph nodes by the clearing method. J Am Coll Surg 1994;178: 223-228.

21. Morisawa E, Yasutomi M, Shindou K et al. Distribution of metastatic lymph nodes in colorectal cancer by the modified clearing method. Dis Colon Rectum 1994;37: 219-223.

\section{ENDEREÇOPARACORRESPONDÊNCIA:}

Dr. Sidney Roberto Nadal

Rua Dr. Virgílio Carvalho Pinto, 381/23

05415-030 — São Paulo-SP 